\title{
Friction-reducing micro/nanoprotrusions on electrodeposited Ni-Co alloy coating surface fabricated by laser direct writing
}

\author{
HAIFENG YANG, TIANCHI CHEN*, JIGUO QIAN, JING HAN, HAIDONG HE, \\ LONGPENG ZHOU, ENLAN ZHAO, WEI TANG and HUA ZHU \\ College of Mechanical \& Electrical Engineering, China University of Mining and Technology, Xu Zhou 221116, P R China
}

MS received 9 November 2013; revised 6 March 2014

\begin{abstract}
Ni-Co coating or texturing surface has been studied extensively to improve the anti-friction ability of the surface. In this paper, we combine the advantages of $\mathrm{Ni}-\mathrm{Co}$ coating and textured surface, and then use a simple, novel and easily controlled method to fabricate a series of micro/nanoprotrusions on the Ni-Co coating surface. The nanotribology properties were characterized by AFM. The result shows that the micro/nanotextured surface significantly reduced the friction forces compared with the original Ni-Co coating surface. The half-ellipsoid patterns have better tribology properties than half-hemispherical patterns. Both laser power and laser scanning speed were found to influence the friction performances.
\end{abstract}

Keywords. Laser direct writing; micro/nanotexured surface; AFM; nanotribology properties.

\section{Introduction}

$\mathrm{Ni}-\mathrm{Co}$ coating is widely used in computer and MEMS fields, such as computer hard drives, ${ }^{1}$ sensors, actuators and inductors, ${ }^{2}$ due to its high strength, magnetic properties and anti-corrosion ability. ${ }^{3,4}$ Therefore, Ni-Co coating is expected to replace the chromium coatings in the automotive, aerospace and other industrial fields because of its better mechanical properties and lower friction performance. ${ }^{5,6}$

Nowadays, with the development of MEMS field, the serious frictional and adhesive problems are increasingly prominent because of the increased surface forces caused by dramatically increased surface-area-to-volume ratio. ${ }^{7}$ Therefore, fabricating the low frictional and adhesion surface is of great importance. ${ }^{8,9}$ The nanotextured surface study, due to its unique properties greatly improved the tribological properties, is attracted by more and more scholars. For instance, Xiaoliang Zhang et $a l^{10}$ had investigated the nano-tribological properities of the texture produced by self-assembling Au nanoparticles (NPs) with diameters of 20 and $50 \mathrm{~nm}$ on the silicon (100) surfaces. The results show that the surface texture can reduce the friction because of the much reduced real contact area. Ying Wang et $a l^{11}$ fabricated nanogrooves patterning in the Si surface by using photolithography and investigated the tribological properties at the nanoscale. They found that tribological performance could be greatly improved by designing suitable surface topography. Mo et $a l^{12,13}$ had fabricated a nanotexture on $\mathrm{H}$-passivated $\mathrm{Si}$ surface by current-induced local anodic oxidation and investigated its nanotribological properties by a colloidal probe. These results showed that the

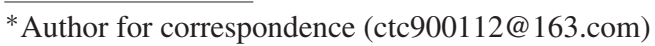

nanotexture can strongly reduce adhesion and friction force at the nanometre scale. Hence, surface texturing is considered as an effective method to decrease the adhesion and friction in magnetic storage and M/NEMS devices. ${ }^{14}$

Due to the good behaviour of the friction performance, fabricating the uniformly distributed and low cost texture is becoming the hotspot in the surface engineering field. In the past, various kinds of methods were applied to produce nanotexture. Photolithography, such as electrobeam lithography, ${ }^{15}$ interference lithography, ${ }^{16}$ imprint lithography ${ }^{17}$ and soft lithography, ${ }^{18}$ is the most common way to fabricate the nanoscale regular patterning. However, the cumbersome process and expensive cost obviously limit the industrial application of photolithography in fabricating nanotexture. Other methods, such as chemical vapour deposition, ${ }^{19} \mathrm{dip}$ coating, ${ }^{8}$ can also produce nano-size textures with the advantage of easy process and low cost. However, the textures obtained from these kinds of methods are randomly distributed uncontrolled nanostructures. The laser direct writing is a cheap and easily operating method to improve surface performance. As the research continues, many researchers find laser direct pulse irradiation can manufacture controllable nanotextures. Ionin et $a l^{20}$ investigated the influence of the incident energy density and the number of pulses on the structured surface topology. Quasiperiodic nano and microstructures have been fabricated on silicon surfaces using 744 and $248 \mathrm{~nm}$ fs-laser pulses. However, according to prior research, there are still some problems for the method of laser direct writing. Laser parameters that can induce micro/nano textures are in a small suitable range and it is difficult to control very precisely. Moreover, local room temperature and humidity have an important impact on experimental final results. 
In this paper, it is concluded that the laser direct writing can be used to fabricate large-area controlled nanotextured surfaces. The surface morphology and microstructure of the laser-processed area on the coating surface was characterized by SEM. The nanotribology properties on the processed area were investigated by atomic force microscopy (AFM).

\section{Experimental}

Stainless steels $(316 \mathrm{~L})$ were used as substrates. The size of all the specimens was $10 \times 15 \mathrm{~mm}$ with thickness of $1.1 \mathrm{~mm}$. All the specimens were first polished, then degreased in acetone under ultrasonic vibration for $5 \mathrm{~min}$, and washed in an $\mathrm{HCl}$ solution $\left(1 \mathrm{~mol} \mathrm{l}^{-1}\right)$ for $2 \mathrm{~min}$ at room temperature for removing the surface oxide layer.

The Ni-Co alloy coatings were fabricated by the method of electrodeposition. The optimal compositions of electroplating bath used in this research were all Grade AR and the formula of plating solution followed was: cobalt sulphate: $20 \mathrm{~g} \mathrm{l}^{-1}$, nickel sulphate: $60 \mathrm{~g} \mathrm{l}^{-1}$, nickel chloride: $30 \mathrm{~g} \mathrm{l}^{-1}$ and boric acid: $30 \mathrm{~g} \mathrm{l}^{-1}$. All the solutions used were freshly prepared with distilled water. The temperature of bath was about $50^{\circ} \mathrm{C}$ and its $\mathrm{pH}$ value was at a controlled range of 3.5 to 4.5 . The electrodeposition was operated in a selfmade electroplating bath. An electrochemical workstation (CHI660D) was used as a power source and a DF-1 heating magnetic whisk was used to control the temperature of electroplating bath and keep all the solutions stirring well.

Then the samples with electrodeposited Ni-Co coatings were processed by the laser direct writing. The schematic diagram of the laser direct writing system is shown in figure 1. An ultraviolet laser (DSH-355-10, Photonics Industries, USA) with working wavelength of $355 \mathrm{~nm}$ was

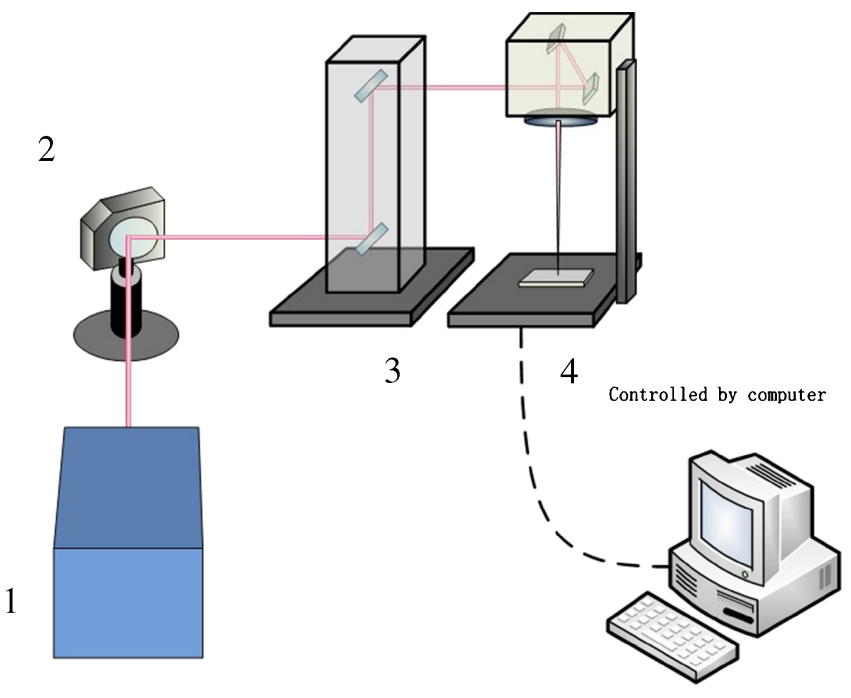

Figure 1. Schematic of the laser direct writing system: (1) laser, (2) mirror, (3) $45^{\circ}$ mirror and (4) laser marker. selected to process the specimen. Mirrors were used to reflect the laser beam incident into a laser marker. The laser marker controlled by computer played a role in controlling the motion of the laser beam. In this experiment, the specimens were placed under the laser marker and irradiated by a laser beam in various laser powers (starting from $32.5 \mathrm{~mA}$, each time increasing $0.05 \mathrm{~mA}$, ending with $35.0 \mathrm{~mA}$ ) with five different scanning velocities $\left(1,3,6,10\right.$ and $\left.20 \mathrm{~mm} \mathrm{~s}^{-1}\right)$ and scan line interval of $0.01 \mathrm{~mm}$, under a focussed laser beam diameter of $30 \mu \mathrm{m}$.

Laser-processed areas were examined by scanning electron microscopy (SEM) to investigate the influence of laser power and scanning velocities on the surface morphology and nanostructure of the coating surface. The nanotribological properties of laser-processed areas were measured by atomic force microscope (AFM). In this experiment, the AFM (CSPM5500 electronics, Benyuan Nano-Instrument, China) used contact mode. Commercially available rectangle $\mathrm{Si}$ cantilever with a normal force constant $0.2 \mathrm{~N} \mathrm{~m}^{-1}$ and a $\mathrm{Si}$ tip with a radius of less than $10 \mathrm{~nm}$ (Budget Sensors Instruments Inc.) was employed. The probe coating with aluminium reflex coating was used to scan the sample at a scanning rate of $1 \mathrm{~Hz}$ and scanning length of $50.0 \mu \mathrm{m}$, while simultaneously measuring the frictional response signal at room temperature and humidity of $30 \%$.

\section{Results and discussion}

\subsection{Morphology of textured Ni-Co alloy coating surfaces}

Figure 2 shows the morphology of Ni-Co alloy coating after laser direct writing in different laser powers from 33.35 to $34.0 \mathrm{~mA}$, at the same scanning speed of $6 \mathrm{~mm} \mathrm{~s}^{-1}$. Figure 3 shows AFM images of Ni-Co coating surface treated by different laser powers. In these series of SEM images, the original morphology of $\mathrm{Ni}-\mathrm{Co}$ alloy coating is shown in figure $2 \mathrm{a}$. A lot of irregular size and random distributed microparticles and nanobumps are observed in figure $3 \mathrm{a}$. The morphology of the surface is roughness with some bumps and grooves. As seen in figure $2 \mathrm{~b}$, after laser direct writing with power of $33.35 \mathrm{~mA}$, the protrusions on the coating have a tendency to become more regular, but the coating surface of laser processing area is still irregular. With the increase of laser power, some half-hemispherical micro/nanoprotrusions are created on the laser processing area at the laser power of $33.40 \mathrm{~mA}$ (figure 2c), and the surface defects are replaced by uniform and regular patterns, as shown in figure 3b. Then, the half-hemispherical micro/nanoprotrusions transform to half-ellipsoid structures when the laser power reaches 33.55 and $33.75 \mathrm{~mA}$, as shown in figure $2 \mathrm{~d}$ and e. These half-ellipsoid micro/nanoprotrusions are evenly arranged on the $\mathrm{Ni}-\mathrm{Co}$ coating surface in figure 3c. As laser power continuously increases, the textures on the surface almost disappear and the whole treated area is under a smooth status after laser writing at the power of $34.0 \mathrm{~mA}$ (figure 2f). Figure 4 shows a histogram 


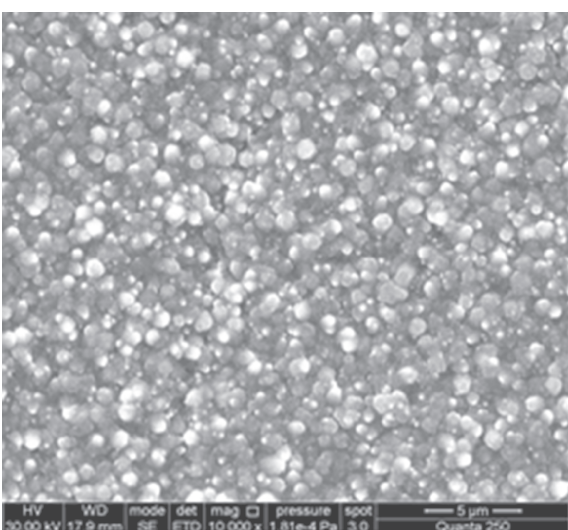

(a)

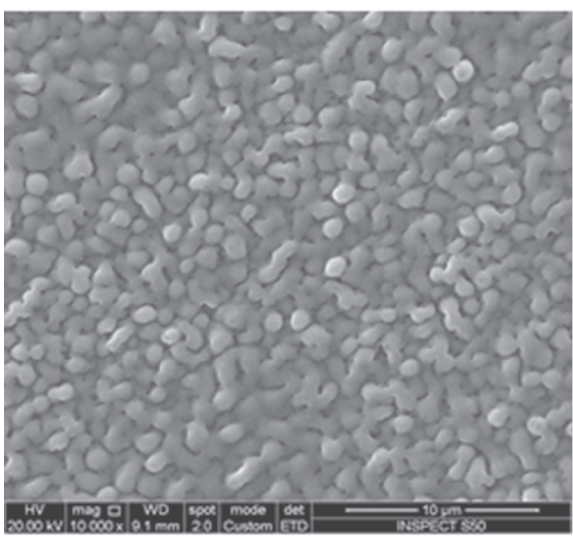

(d)

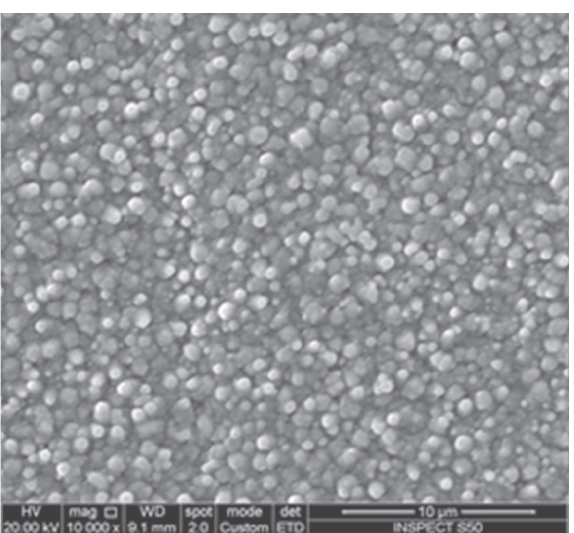

(b)

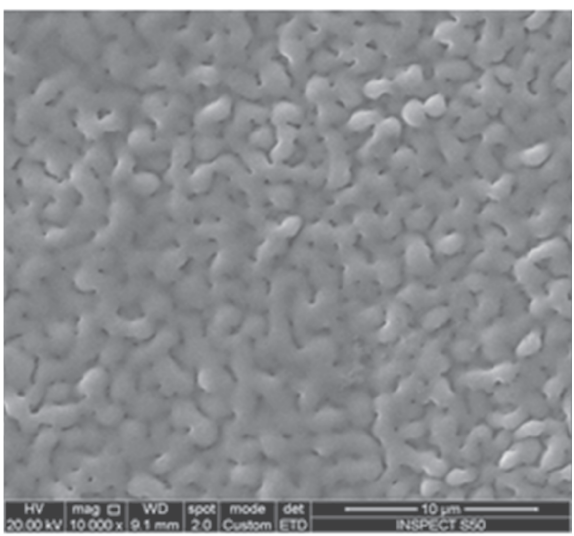

(e)

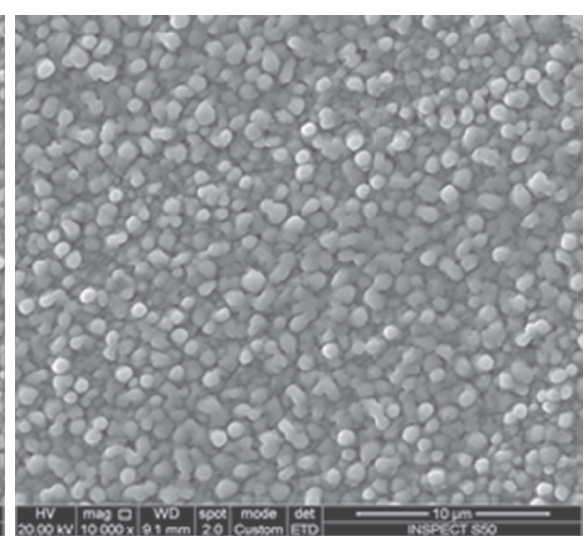

(c)

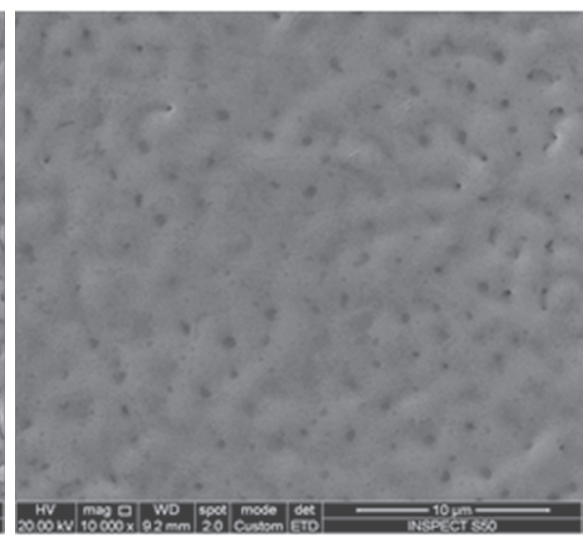

(f)

Figure 2. SEM images of (a) Ni-Co alloy coating surface before laser-beam treatment (unprocessed coating surface) and (b-f) the processing region under laser treatment at laser powers of 33.35, 33.40, 33.55, 33.75 and $34.0 \mathrm{~mA}$, respectively.

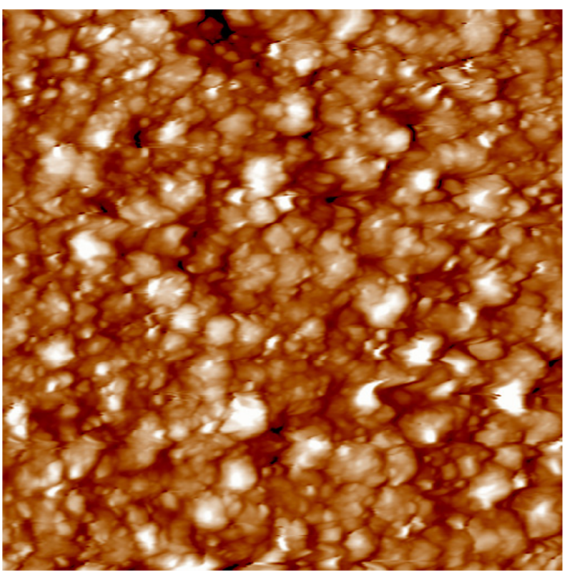

(a)

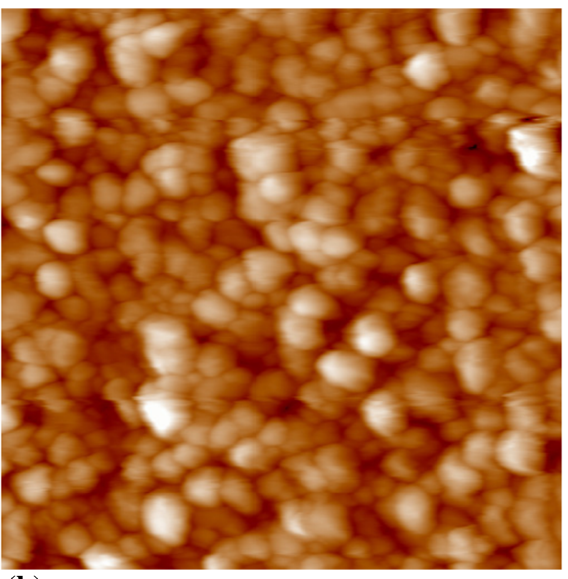

(b)

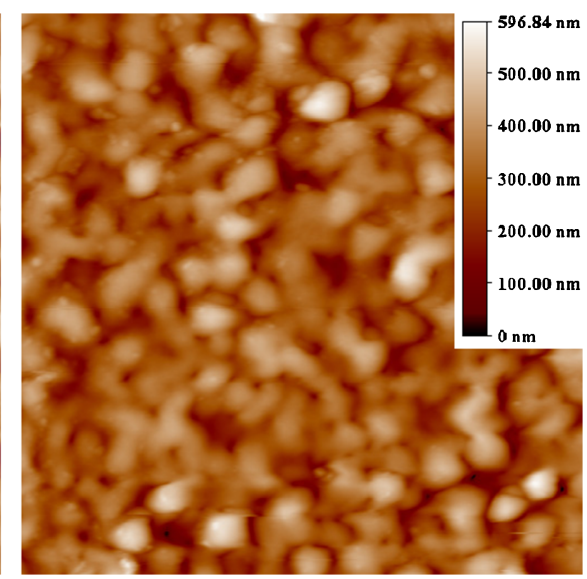

(c)

Figure 3. AFM images of different micro/nanoprotrusions on the Ni-Co coating surface induced by different laser powers: (a) unprocessed, (b) $33.40 \mathrm{~mA}$ and (c) $33.55 \mathrm{~mA}$.

that visually demonstrates the average surface roughness of $\mathrm{Ni}-\mathrm{Co}$ coating surface processed by different laser powers at a fixed laser scanning speed of $6 \mathrm{~mm} \mathrm{~s}^{-1}$. The histogram demonstrates that the average surface roughness decreases with the increase in laser power. Unprocessed Ni-Co coating surface has the biggest average surface roughness. The surface quality is improved when the laser powers are increased. The histogram of the average surface roughness of Ni-Co coating surface processed by different laser powers can also supply some information about the formation 


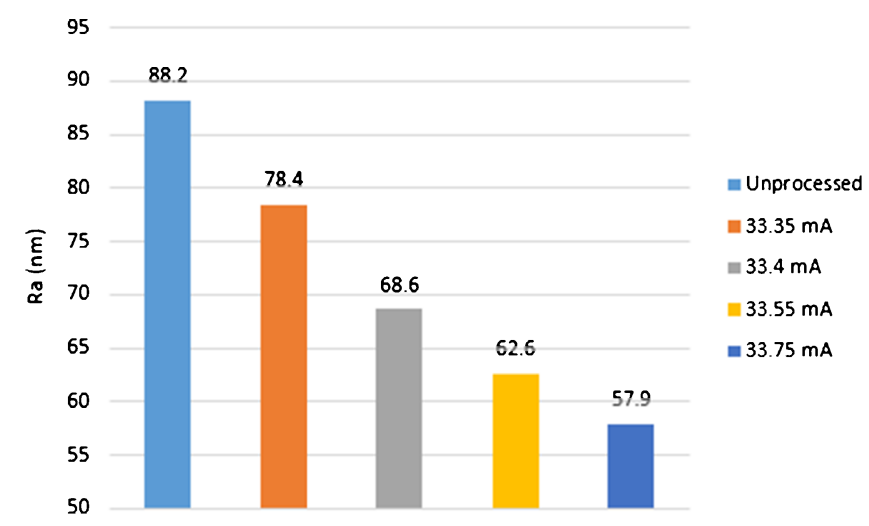

Figure 4. Histogram of the average surface roughness of Ni-Co coating surface processed by different laser powers at a fixed laser scanning speed of $6 \mathrm{~mm} \mathrm{~s}^{-1}$.

mechanism of micro/nanotextures induced by laser direct writing on the Ni-Co coating surface. Therefore, laser power is one of the key parameters to fabricate micro/nanotextured surface. The uniform half-hemispherical and half-ellipsoid micro/nanoprotrusions can be obtained by adjusting appropriate laser powers.

The formation mechanism of nanotextures induced by laser direct writing on the Ni-Co surface has not been thoroughly researched. However, we can reference the formation mechanism of nanostructure or nanogratings induced by pulse-laser ablation, which has been researched by many scholars. ${ }^{21,22}$ The formation of micro/nanoprotrusions may be concluded by an initial non-uniform scattered laser energy deposition and melting in the nanoscale. ${ }^{23}$ The bump-like surface has well-defined thresholds. ${ }^{24}$ With the increase of laser power, the incident laser fluence is close to the laser ablation threshold. When the incident laser fluence is above the melting threshold, the micro/nanoprotrusions on the $\mathrm{Ni}-\mathrm{Co}$ coating surface become possible. Because of initial non-uniform scattered laser energy deposition, not all irradiation regions of the laser beam reach the melting threshold. The area in which laser energy is above the melting threshold begins melting in the nanoscale. Then, a high radial temperature gradient in a nanomelt can induce a radial surface tension gradient that expels the liquid to the periphery of the nanomelt. ${ }^{24}$ The solidification of the molten metal starts from the edge of molten liquid and soon reaches the centre of molten liquid. ${ }^{23}$ It can also be responsible for the formation of nanobumps due to fast freezing of the expelled liquid on the boundary with the solid state material. This will explain the formation of half-hemispherical micro/nanoprotrusions when the laser power reaches $33.40 \mathrm{~mA}$, as shown in figure 2c. When the laser power continuously increases to $33.55 \mathrm{~mA}$, the number of nanomelting areas increases, with many of them overlapping. So, half-ellipsoid nanoprotrusions form on the laser-processed area. As the laser power reaches $33.75 \mathrm{~mA}$, more half-ellipsoid nanoprotrusions fuse together, becoming microbumps, as shown in figure $2 \mathrm{e}$. When laser power is above $34.0 \mathrm{~mA}$, the laser energy exceeds the laser ablation threshold. The process becomes unstable and micro/nanoprotrusions are destroyed by high focus laser energy. The target surface begins to remelt. The treated surface becomes smooth, as shown in figure $2 \mathrm{f}$.

To investigate the relationship between laser scanning speed and surface morphology, different laser scanning speeds $\left(1,3,6,10,20 \mathrm{~mm} \mathrm{~s}^{-1}\right)$ were used to process $\mathrm{Ni}-\mathrm{Co}$ coating surface at the same laser power. As shown in figure 5a, a series of SEM images shows half-hemispherical micro/nanoprotrusions on the Ni-Co coating surfaces fabricated by different scanning speeds $\left(1,10,20 \mathrm{~mm} \mathrm{~s}^{-1}\right)$ when the laser power is $33.40 \mathrm{~mA}$. It can be clearly observed that the half-hemispherical micro/nanoprotrusions on the Ni-Co coating surface are not of the same sizes. With the increasing of laser scanning speed, the size of half-hemispherical micro/nanoprotrusions decreases. What's more, the distribution of micro/nanoprotrusions becomes uniform when laser scanning speed increases. The same law applies when laser power is $33.55 \mathrm{~mA}$, as shown in figure 6. It means that the sizes and distributions of half-ellipsoid micro/nanoprotrusions on the Ni-Co coating surface are related to laser scanning speed. When the laser scanning speed becomes faster, the half-ellipsoid micro/nanoprotrusions grow bigger. The average surface roughness of $\mathrm{Ni}-\mathrm{Co}$ coating surface treated by different laser scanning speeds at a fixed laser power of $33.40 \mathrm{~mA}$ is measured by AFM. These roughness data are presented in a histogram, as shown in figure 7. It can be observed that the average roughness first decreases and then increases with the increasing of laser scanning speed. The roughness in the laser-treated area, no matter what laser powers or laser scanning speeds are set, is still smaller than that in the unprocessed area. Hence, the sizes of half-hemispherical micro/nanoprotrusions and half-ellipsoid micro/nanoprotrusions can be precisely controlled by adjusting different laser scanning speeds.

The formation mechanism of this phenomenon is mainly caused by the grain growth and crystallization. ${ }^{24}$ The grain growth is influenced by temperature, energy and cooling rates. Laser energy density is mainly used to measure the laser energy on the processed surface. Laser energy density can be explained by the following formula:

$$
E s=F /(V \cdot D),
$$

where $F$ is the laser intensity, $V$ is the scan velocities and $D$ is the laser spot diameter. Hence, in the condition of same laser intensity and laser spot diameter, as laser scanning velocity increases, the laser energy density in each scanning trace is reduced. Lower laser energy means less heat can be absorbed by the substrate. Lower scanning velocity, corresponding to higher temperature and longer interaction time, results in higher degree of crystallinity and larger size of micro/nanoprotrusions. ${ }^{25}$ The higher scanning velocity, implying less instantaneous absorbed energy and shorter 


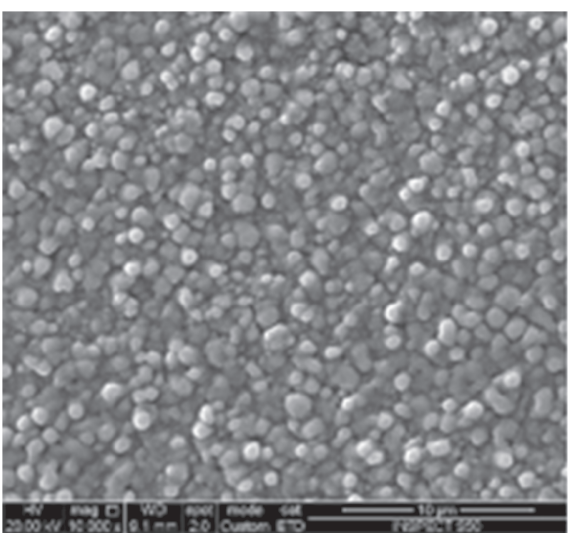

(a)

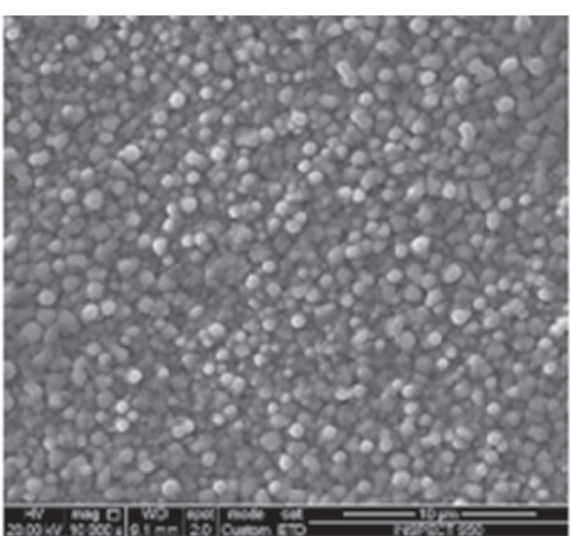

(b)

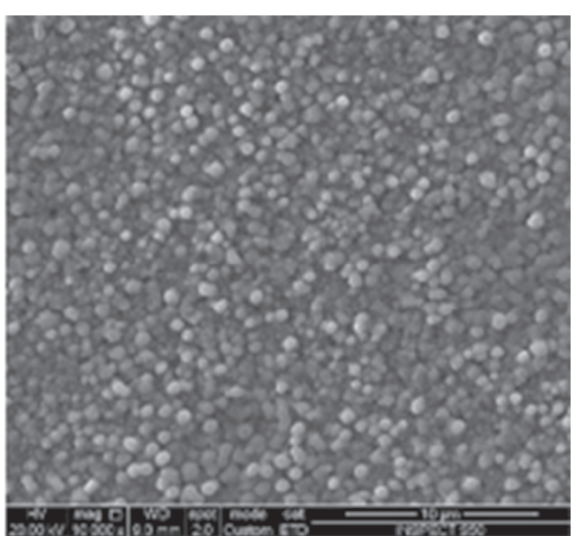

(c)

Figure 5. SEM images of half-hemispherical textured $\mathrm{Ni}-\mathrm{Co}$ alloy coating surface at different scanning speeds: (a) 1 , (b) 10 and (c) $20 \mathrm{~m} \mathrm{~s}^{-1}$.

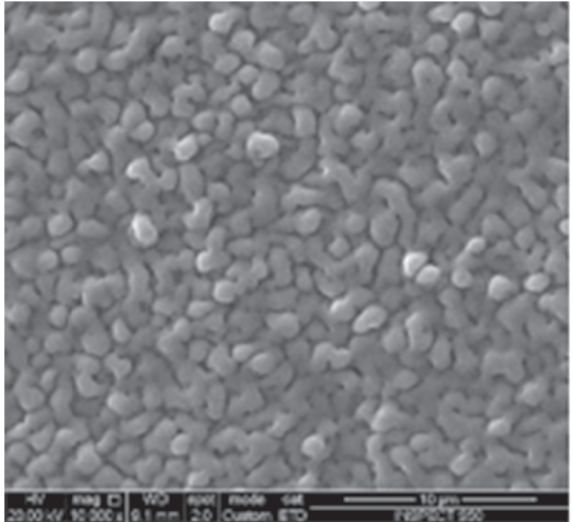

(a)

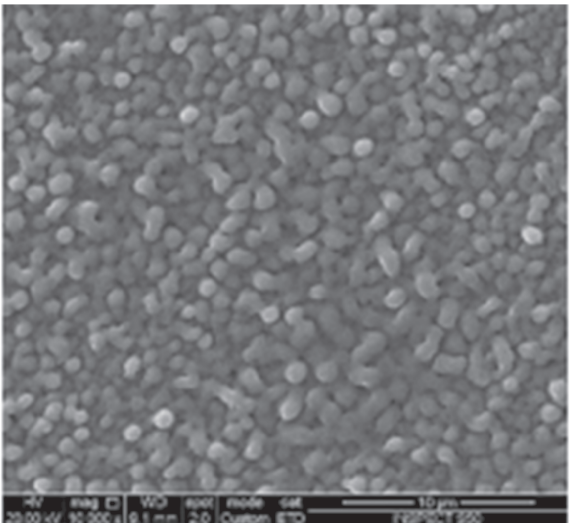

(b)

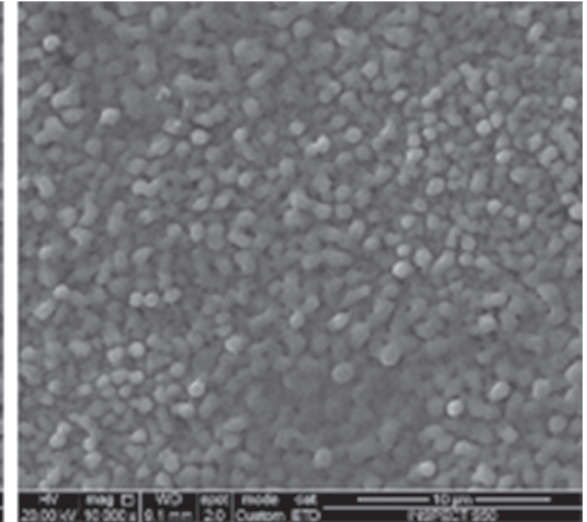

(c)

Figure 6. SEM images of half-ellipsoid textured Ni-Co alloy coating surface at different scanning speeds: (a) 1 , (b) 10 and (c) $20 \mathrm{~m} \mathrm{~s}{ }^{-1}$.

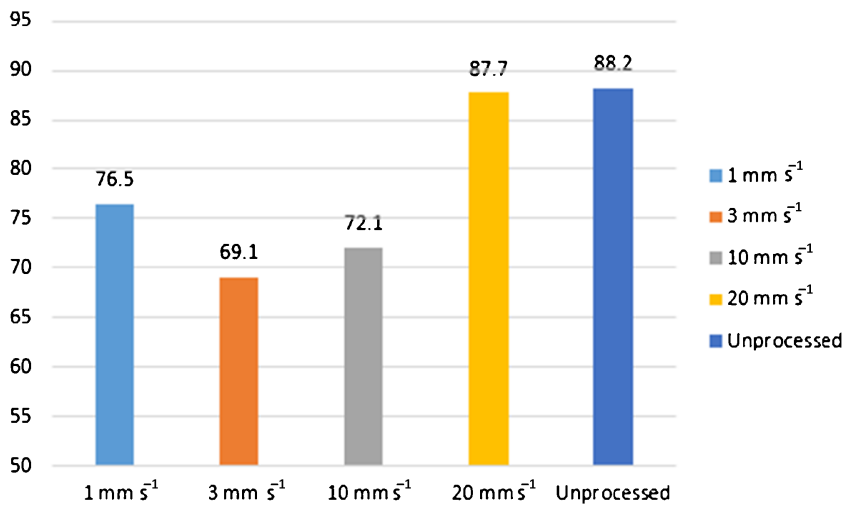

Figure 7. Histogram of the average surface roughness of Ni-Co coating surface processed by different laser scanning speeds at a fixed laser power of $33.40 \mathrm{~mA}$.

interaction time, leads to the incomplete crystallization and smaller size of micro/nanoprotrusions on the surface.

\subsection{Nanotribology properties of the textured $\mathrm{Ni}-\mathrm{Co}$ alloy coating surfaces}

The AFM is used to measure the nanotribology properties of the treated surface. A schematic illustration of how AFM detects normal and lateral force is shown in the inset of figure $8 .^{26}$ With a four-segment photodiode, the twodimensional motion of the reflected beam is measured. Therefore, normal and lateral forces can be detected simultaneously. In the scanning process, bending of the cantilever due to a normal force causes a vertical motion of the reflected beam and torsion of the cantilever due to a lateral force causes a horizontal motion.

Figure 8 shows the friction force of areas processed with different laser powers $(0,33.35,33.40,33.55,33.75 \mathrm{~mA})$ at the laser scanning speed of $6 \mathrm{~mm} \mathrm{~s}^{-1}$ and at different loads, from 0 to $0.8 \mathrm{~V}$. The friction force was given here in the form of voltage signal, which should be proportional to the real friction force. ${ }^{27}$ To obtain precise friction force data, each data point is an average of 5 times 


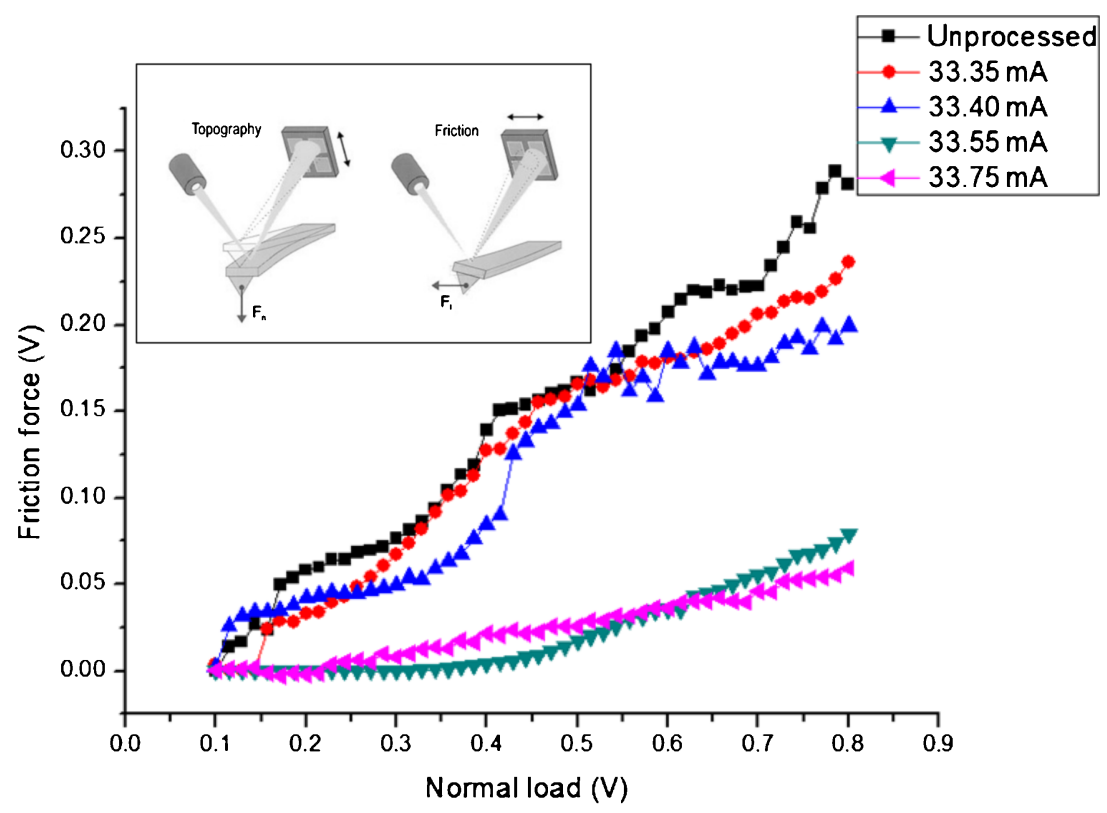

Figure 8. Plots of friction force vs. load curves for the textured Ni-Co coating surface with different laser powers. Inset: Schematic diagram of AFM measures of surface normal and lateral force.

experiments under the same conditions. It can be seen from figure 8 that the friction force increases linearly with loads. This changing trend of friction forces for all areas is roughly the same when the loads increase. However, friction force decreases as the laser power increases. In other words, the friction force is varied by the surface morphology of the $\mathrm{Ni}-\mathrm{Co}$ coating. The friction force on the unprocessed region is bigger than that on the textured region treated by laser in the power of 33.40 or $33.55 \mathrm{~mA}$. Half-ellipsoid textured surface has the smallest friction force among all the laser-processed regions when the laser power is $33.55 \mathrm{~mA}$.

In general, two important factors that control friction at nanoscale in dry/wet contacts are the real area of contact and surface chemistry. ${ }^{28,29}$ In this work, the samples have the same surface chemistry characters. The real area of contact is the crucial factor to influence friction force. According to the fundamental law of interface friction given by Bowdon and Tabor, ${ }^{30}$ for a single asperity contact, friction force depending on the real area of contact can be described as

$$
F_{\mathrm{f}}=\tau A,
$$

where $\tau$ is the shear strength and $A$ is the real area of contact. In order to explain the nanotribology properties between the unprocessed surface and the textured $\mathrm{Ni}-\mathrm{Co}$ alloy coating surfaces, a series of schematic diagrams of the contact between the AFM tip and textured surfaces are shown in figure 9. When the AFM tip is sliding over a protrusion, at the ascending edge, the surface slope is positive, and it is negative at the opposite edge. The measured friction force is higher at the ascending edge because of the additional torsion of the cantilever beam produced by the collision between the tip and the protrusion. However, at the descending edge, no collision effect produces additional torsion. Moreover, an increase in the amount of friction force experienced by the tip at the ascending edge of a protrusion is more than the decrease in the amount of friction force experienced at the descending edge. ${ }^{31}$ Because the tip with radius $10 \mathrm{~nm}$ is too small, it is inevitable to generate collision effect in the process of measuring the friction. As shown in figure 9(a), It is a schematic diagram of racket model proposed by Ruan and Bhushan $^{32}$, where $P$ is represented as normal load, $F$ is represented as friction force, $N$ and $S$ are normal and lateral forces of the protrusion surface separately. $\theta$ is the oblique angle on the protrusion surface. After force analysis and calculation, the force at the ascending edge can be described as:

$$
F=P\left(\mu_{0}+\tan \theta\right)
$$

and the force at the descending egde as:

$$
F=P\left(\mu_{0}-\tan \theta\right) .
$$

When a tip is sliding over a protrusion at the ascending edge, it is positive, and, at the opposite edge, it is negative. So, the measured friction force is increased with $\tan \theta$ increasing, which is equivalent to the increase of the slope on the protrusion surface. Figure $9 \mathrm{~b}$ shows schematic diagrams of the contact between the probe and unprocessed surface. Figure 9c shows schematic diagrams of the contact between the probe and surface with half-hemispherical micro/nanoprotrusions. Figure 9d shows schematic diagrams of the contact between the probe and surface with halfellipsoid micro/nanoprotrusions. There are a lot of irregular sizes and random distributed particles on the unprocessed surface. The collision effect is the most obvious 


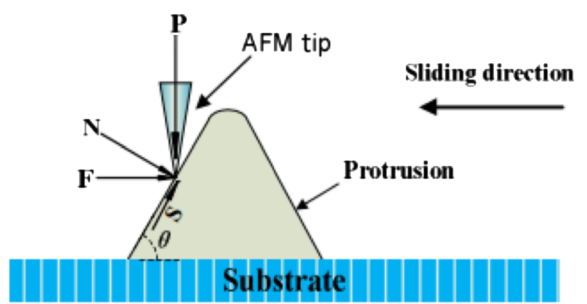

(a)

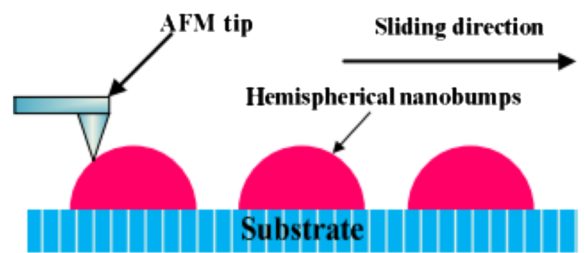

(c)

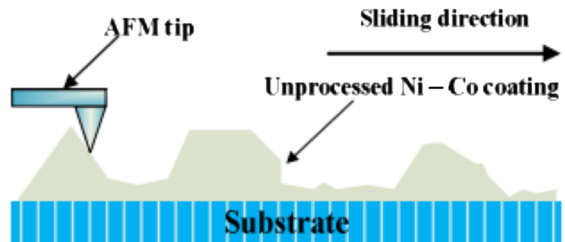

(b)

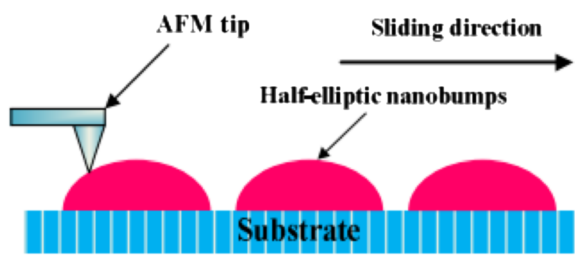

(d)

Figure 9. Schematic diagram of contact between AFM tip and different surface conditions. (a) The force analysis of racket model, (b) the contact between AFM tips and unprocessed surface, (c) the contact between AFM tips and hemisperical nanobumps surface and (d) the contact between AFM tips and hemisperical nanobumps surface.
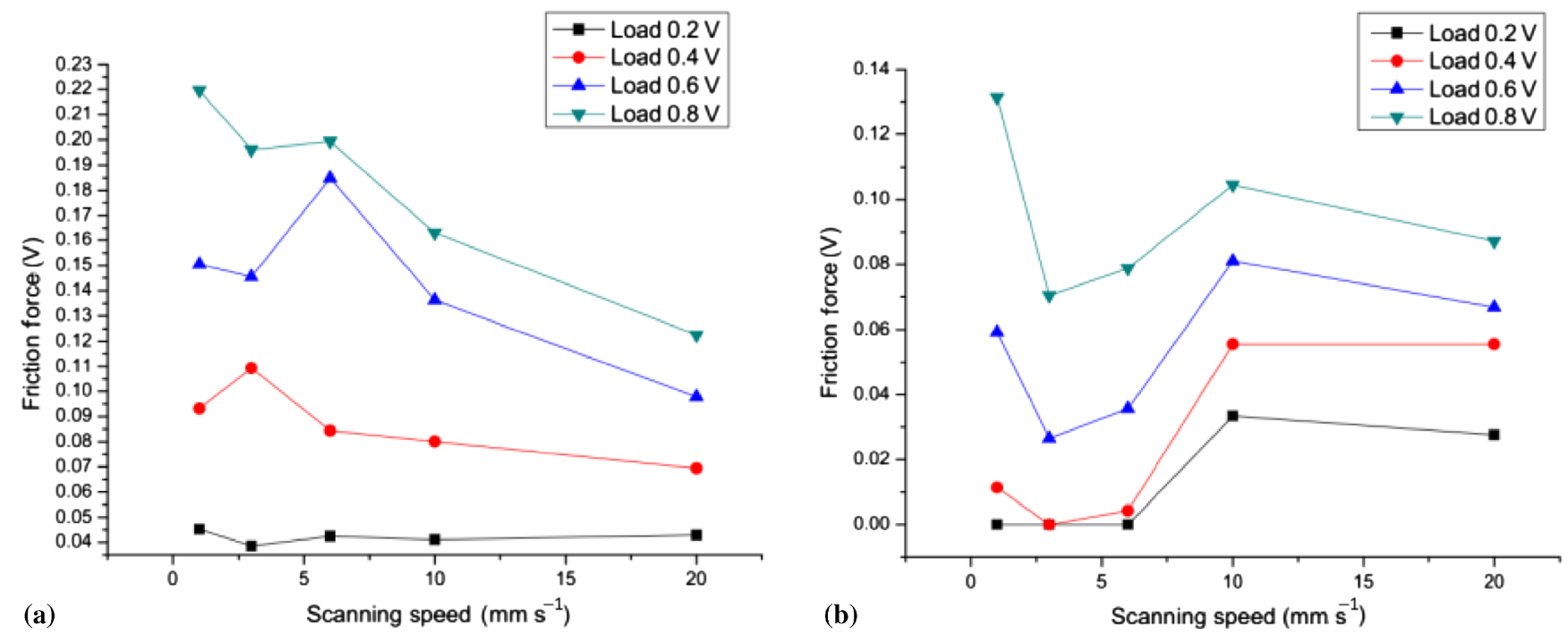

Figure 10. Plots of friction force vs. laser scanning speed curves for (a) hemispherical textured Ni-Co alloy coating surface and (b) half-ellipsoid textured $\mathrm{Ni}-\mathrm{Co}$ alloy coating surface with constant normal load of $0.2,0.4,0.6$ and $0.8 \mathrm{~V}$.

and the average slope is the biggest compared with the other two textured surfaces. Hence, the friction is the biggest on the unprocessed surface. A comparison between half-hemispherical micro/nanoprotrusions and half-ellipsoid micro/nanoprotrusions is presented in figure $9 \mathrm{c}$ and $\mathrm{d}$. The half-ellipsoid micro/nanoprotrusions with smaller slope and intervals among micro/nanoprotrusions are acquired smaller friction force than half-hemispherical micro/nanoprotrusions. In addition, the real area of contact $^{27}$ between the AFM tip and textured surface is remarkably reduced leading to an obvious descent in the frictional force.

Figure 10 shows the relationship between the friction force and the different scanning speeds at loads of $0.2,0.4,0.6$ and $0.8 \mathrm{~V}$. Figure 10a shows the change of the friction force measured from half-hemispherical textured surface with different scanning speeds $\left(1,3,6,10\right.$ and $\left.20 \mathrm{~mm} \mathrm{~s}^{-1}\right)$ at loads of $0.2,0.4,0.6$ and $0.8 \mathrm{~V}$. It can be seen that the friction force decreases with the increase in scanning speed. As mentioned in this paper, the size of micro/nanoprotrusions is influenced by the laser scanning speed. The faster scanning speed, corresponding to the smaller size of the halfhemispherical micro/nanoprotrusions and intervals among micro/nanoprotrusions, results in less additional torsion of the cantilever beam produced by the collision effect ${ }^{31}$ and less real contact area, when the probe is sliding on the surface with half-hemispherical micro/nanoprotrusions. With 
the increase of the load, this rule becomes more obvious. Due to the friction force varying obviously with the load increased, the difference of friction force among different laser scanning speeds becomes obvious. As seen from figure $10 \mathrm{~b}$, the friction force on the half-ellipsoid textured surface first decreases with the laser scanning velocity increasing. However, when the scanning velocity exceeds $3 \mathrm{~mm} \mathrm{~s}^{-1}$, the friction force increases with the laser scanning velocity increasing. The smaller size of micro/nanobump resulting in smaller real contact area, grain growth in the low scanning speed is considered as another main reason to respond for changes in the friction force. In a low laser scanning speed, the micro/nanobump grew bigger and nanoscale grain grew by high temperature and long interaction time on the micro/nanoprotrusions, increasing the additional torsion of cantilever beam, ${ }^{33}$ leading to an increase of the friction force. With laser scan speed increasing, the surface becomes smooth and the friction force conveniently decreases. However in the high speed, when the scanning speed exceeds $3 \mathrm{~mm} \mathrm{~s}^{-1}$, the surface becomes rough, as shown in figure 7 , because of the incomplete crystallization. ${ }^{25}$ This is the main reason for the increase of the friction force in this period. Hence, the optimal scanning speed of $3 \mathrm{~mm} \mathrm{~s}^{-1}$ is acquired by a comparison of the friction forces in different scan speeds.

\section{Conclusions}

In the present research, the morphology of the textured $\mathrm{Ni}-\mathrm{Co}$ coating surface is fabricated by laser direct writing and its nanotribology properties have been investigated. The following conclusions could be drawn:

(1) The micro/nanotextured surface can be fabricated by laser direct writing. By adjusting appropriate laser power $(33.40,33.55 \mathrm{~mA})$, half-hemispherical and halfellipsoid micro/nanoprotrusions can be obtained on the coating surface. The laser scan speed can control the size of micro/nanobump. The scanning speed is faster, and the size of micro/nanoprotrusions is smaller.

(2) The nanotribology properties of the textured surface have been studied. It is found that the friction force is related to laser powers. The friction force on the textured surface is smaller than the unprocessed surface. The friction force on the half-hemispherical textured surface is greater than half-ellipsoid textured surface. This conclusion also proves that the textured surface can reduce the friction.

(3) The friction force on the textured surface has a great relationship with laser scanning speed. The laws between friction force and scanning speed are not the same on different textured surfaces. As comprehensive analysis of the friction force data, the optimal scanning speed, $3 \mathrm{~mm} \mathrm{~s}^{-1}$, is picked.

\section{Acknowledgement}

We gratefully acknowledge the Natural Science Foundation of China (51105360), the Natural Science Foundation of Jiangsu Province (BK2011218), Research Fund for the Doctoral Program of Higher Education of China (20120095120014) and the Fundamental Research Funds for the Central Universities under Grant (2012QNA25) for supporting this work.

\section{References}

1. Shi L, Sun C F, Gao P, Zhou F and Liu W M 2006 Surf. Coat. Technol. $\mathbf{2 0 0} 4870$

2. Golodnitsky D, Rosenberg Y and Ulus A 2002 Electrochim. Acta. $\mathbf{4 7} 2707$

3. Srivastava M, Selvi V E, Grips V K W and Rajam K S 2006 Surf. Coat. Technol. 2013051

4. Kim D, Park D Y, Yoo B Y, Sumodjo P T A and Myung N V 2003 Electrochim. Acta. $\mathbf{4 8} 819$

5. Wang L, Gao Y, Xue Q, Liu H and Xu T 2005 Appl. Surf. Sci. 242326

6. Ranjith B and Paruthima Kalaignan G 2010 Appl. Surf. Sci. 25742

7. Hsu S M 2004 Tribol. Int. 7537

8. Zou M, Cai L and Wang H 2006a Tribol. Lett. 2125

9. Zou M, Cai L, Wang H and Xu J 2006b Solid-State Lett. 9 G31

10. Xiaoliang Zhang, Xiaohua Zhong and Xu Meng et al 2012a Tribol. Lett. 4665

11. Ying Wang, Liping Wang and Qunji Xue et al 2010 Colloid Surface A $\mathbf{3 7 2} 139$

12. Mo Y, Zhao W, Huang D, Zhao F and Bai M 2009a Ultramicroscopy 109247

13. Mo Y, Wang Y, Pu J and Bai M 2009b Langmuir 2540

14. Etsion I 2004 Tribol. Lett. 17733

15. Di F, Enzo M, Cojoc D et al 2003 Proc. SPIE 5225113

16. Murillo R, Van W H A, Abelmann L and Lodder J C 2005 Microelectron. Eng. 26078

17. Kono Y, Sekiguchi A and Hirai Y 2005 Proc. SPIE 5753912

18. Yao J, Yan X, Lu G, Zhang K, Chen X, Jiang L and Yang B 2004 Adv. Mater. 1681

19. Singh D P, Singh A K and Srivastava O N 2003 J. Nanosci. Nanotechnol. 3545

20. Ionin A A, Kudryashov S I and Makarov S V et al 2011 Quant. Electron 41829

21. Zhou G S, Fauchet P M and Siegman A E 1982 Phys. Rev. B 265366

22. Shen M Y, Crouch C H, Carey J E et al 2003 Appl. Phys. Lett. 821715

23. Vorobyev A Y and Chunlei Guo 2006 Opt. Express 142164

24. Koch J, Korte F, Bauer T, Fallnich C, Ostendorf A and Chichkov B N 2005 Appl. Phys. A 81325

25. Liu H, Viejo F, Guo R X, Glenday S and Liu Z 2010 Surf. Coat. Technol. 2041549

26. Bhushan B 2001 Modern tribology handbook (New York: CR Press) Vol. 305 
27. Song S Y, Ren S L, Wang J Q, Yang S R and Zhang J Y 2006 Langmuir 226010

28. Jung Y C and Bhushan B 2006 Nanotechnology 17 4970

29. Wenjie Zhao, Liping Wang and Qunji Xue 2010 Colloid Surface A 366191
30. Bowdon F P and Tabor D 1967 Friction and lubrication (London Methuen)

31. Bhushan B 2005 Wear 2591507

32. Ruan J and Bhushan B 1994 J. Appl. Phys. 765022

33. Xiaoliang Zhang, Yongjuan Lu, Eryong Liu et al 2012b Colloid Surface A 40190 